\title{
VLBI observations of spacecraft
}

lan Avruch*, S. V. Pogrebenko, and L. I. Gurvits

Joint Institute for VLBI in Europe

E-mail: avruchejive.nl pogrebenkodjive.nl lgurvitsejive.n

Since the launch of Sputnik in 1957, spacecraft have been routinely tracked within the Solar System by radio telescope. An interferometric technique known as Delta-DOR determines positions of spacecraft with milliarcsecond precision. We have been developing a technique for doing VLBI on spacecraft signals, which differ importantly from natural sources. Such a capability could provide relative astrometric precision at the microarcsecond level. We will describe the technique and present results obtained from observations of the Huygens probe landing on Titan (January 2005) and the impact of the SMART-1 spacecraft on the Moon (September 2006).

The 8th European VLBI Network Symposium

September 26-29, 2006

Toruń, Poland

* Speaker. 


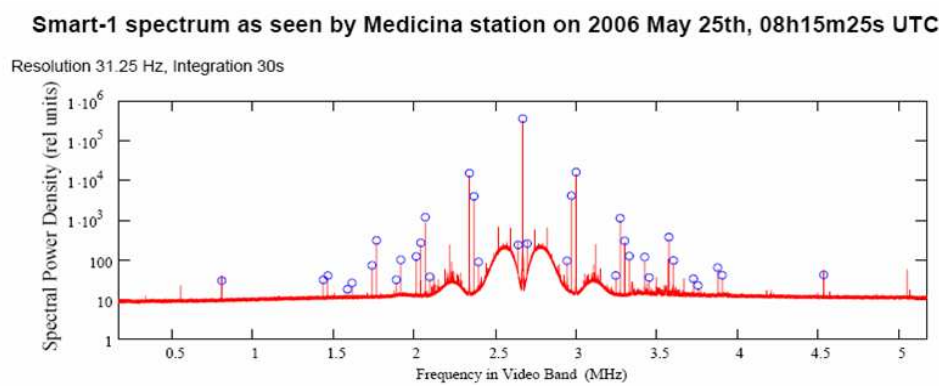

Figure 1: An example spectrum of the signals from ESA's SMART-1 spacecraft. The subcarrier lines, the narrow features spread across the spectrum, are phase-coherent with the carrier line in the centre.

\section{Spacecraft tracking}

Tracking the state vector of a spacecraft is interesting not only for navigation, but also for conducting scientific experiments. For example, measuring the properties of planetary atmospheres through effects on the spacecraft signal during occultation, or testing General Relativity by measuring frequency deviations of the downlink carrier tone as the spacecraft moves near the Sun [1]. In a recent experiment of the latter type, post-fit frequency residuals were very low: below $0.5 \mathrm{mHz}$.

Spacecraft have very constrained power budgets, and although a large craft like the Cassini orbiter has perhaps $20 \mathrm{~W}$ of radiating power and a high-gain parabolic antenna, the Huygens probe, designed to be received by Cassini at ranges less than $10^{5} \mathrm{~km}$, had only $10 \mathrm{~W}$ with a low-gain antenna. Nevertheless, as the carrier tones are very pure, the spectral density is extremely high.

The signals broadcast by spacecraft are confined to allocated frequencies in S,X, and Ka bands. A pure carrier tone is phase-modulated to produce a subcarrier line, which is in turn modulated to carry telemetry data [2]. Figure 11 shows an example spectrum taken from an observation of the SMART-1 spacecraft. The various tones are found to be phase-coherent with the carrier tone.

The frequency stability of the carrier line is key to its use in navigation or radio science experiments. The greatest stability is achieved with a "two-way" mode in which the spacecraft downlink tone is locked to the uplink signal from the ground station, which is in turn locked to an atomic clock. The motion of the ground station with respect to some frame of reference, for example the solar system barycentre, is known and hence the residual Doppler shift of the received signal is a measurement of the spacecraft motion.

For times when two-way communication is impossible, for instance egress from occultation, an on-board oscillator may be used. Ultra-stable oscillators (USOs) in temperature-controlled housings can achieve Allan variances of $10^{-13}$, compared to $10^{-15}-10^{-16}$ for hydrogen masers.

The spacecraft state vector can be determined by measuring range and radial Doppler, and its sky coordinates and angular motion. The first two are straightforward: using a two-way coherent link, the Doppler shift is measured to $1 \mathrm{~Hz}$, and the range or light-time is determined by uplinking a coded pulse, whose round-trip time minus the known instrumental turn-around time within the spacecraft gives the desired result.

Two telescopes with different lines of sight to the spacecraft can simultaneously measure Doppler shifts, and the difference can be used to compute the spacecraft position and velocity. 
This Differenced Doppler technique can measure target velocities to $0.01 \mathrm{~mm} / \mathrm{s}$, and provide an angular position to several-mas-precision [2].

Correlation of the spacecraft signal received at two stations can give the range difference from the spacecraft to the two telescopes. This interferometric measurement of the group delay is called, in this context, Differential One-Way Ranging. Further, the two telescopes can do phasereferencing VLBI on the spacecraft, and this is known as Delta Differential One-Way Ranging ( $\triangle \mathrm{DOR}$ ) [3]. The situation is familiar: a strong quasar is used as reference source, and the two telescopes nod between it and the target spacecraft. An observation typically lasts one hour, with a scan length of five to ten minutes. Only the group delay is measured, with a precision of about $0.15 \mathrm{~ns}$, which is sufficient for the purposes of navigation support.

A VLBI phase delay measurement could be 10 times as precise, given the nature of the signals, and this could be an interesting tool for physical measurements. The $\triangle \mathrm{DOR}$ service provided by DSN and ESA tracking stations for space missions, using dedicated hardware, is geared toward reliability. VLBI science experiments can achieve higher precision, accepting the burden of more complex data reduction and a chance of failure.

\section{Observations of the SMART-1 probe}

ESA's SMART-1 probe went into lunar orbit in November 2004, conducting science observations of the Moon until 3 September 2006, when it finished its mission with a planned impact on the lunar surface. There was scientific rationale for the impact: for example, learning about the properties of surface dust by spectrometry of the flash. The exact time of impact was also of interest, which comes easily from inspection of the recorded data and the station GPS log. We observed the spacecraft in the months leading up to impact and during the event, using it as a test case for future spacecraft experiments. These were not astrometric observations: we did not intend to measure the position of SMART-1. Rather, we were interested in testing reduction algorithms for the strong signal regime.

Figure 2 presents the phase of a subcarrier tone measured at two telescopes as the spacecraft emerges from behind the Moon, and the correlated signal. The slow variation in the visibility phase is due to the lack of phase-referencing corrections. Removing it leaves a residual of $4.6 \mathrm{ps}$ RMS at $40 \mathrm{~ms}$ coherent averaging, the intrinsic precision of the measurement.

\section{Observations of the Huygens probe}

The Huygens probe was launched from the Cassini spacecraft on 25 December 2004 and entered the atmosphere of Titan on 14 January 2005. After initial deceleration by the heat shield, the main parachute opened and the instruments were exposed. The parachute was replaced with a smaller stabilizer chute after 15 minutes, allowing the probe to land within the estimated battery lifetime. After descending for $2^{\mathrm{h}} 27^{\mathrm{m}}$, the probe impacted the surface at about $5 \mathrm{~m} / \mathrm{s}$. Cassini continued to receive telemetry from Huygens for $1{ }^{\mathrm{h}} 12^{\mathrm{m}}$; the VLBI array observed the probe's $2040 \mathrm{MHz}$ carrier for an additional $2^{\mathrm{h}} 3^{\mathrm{m}}$, when the VLBI schedule ended. The batteries are believed to have died 17 minutes later [沛. 

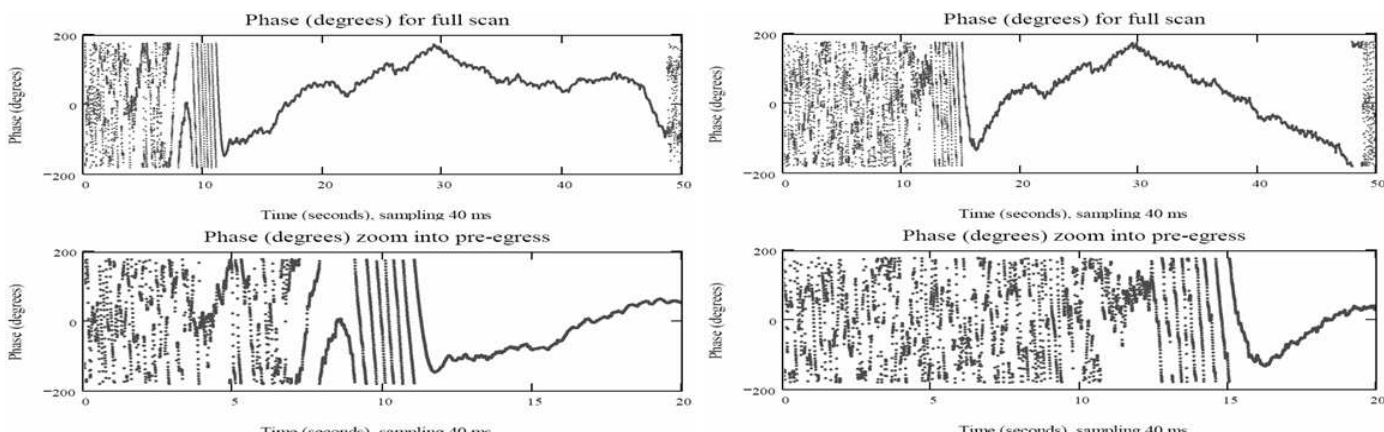

Figure 2: The phase of the SMART-1 spacecraft carrier tone during egress from occultation by the Moon. Consider this the phase of the sine wave voltage detected at each telescope. The diffraction of the signal by the Moon's limb is clear. left: Medicina (Mc), right: Metsähovi (Mh).

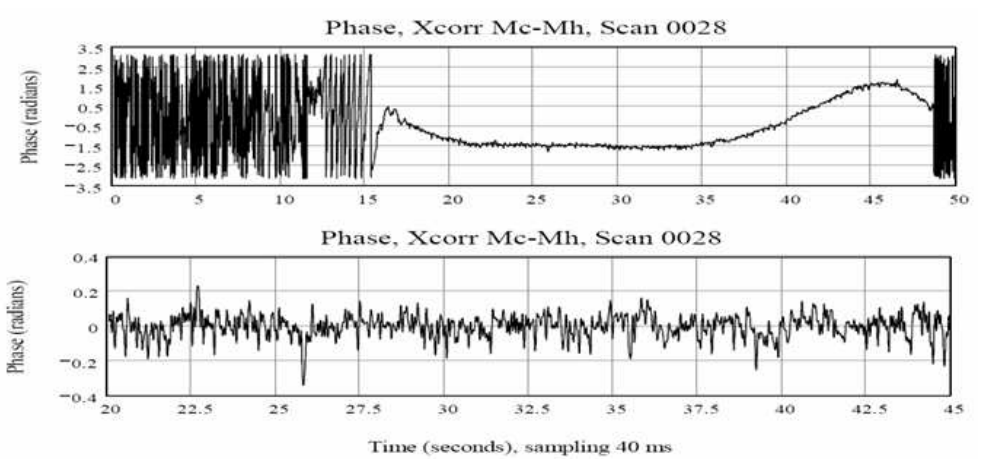

Figure 3: Correlation of the SMART-1 signals, Mc-Mh. The phase, being uncalibrated, is uninterpretable, but the short-timescale noise of $4.6 \mathrm{ps}$ rms is indicative of the potential delay precision.

Planning for the VLBI observations began in late 2003 [5]. There were some unfortunate observing constraints: the celestial location of Titan during the event was not chosen with respect to VLBI reference sources, so a survey had to be undertaken; the Huygens carrier line was outside normal S-band range, so some loss of SNR was inevitable; landing was expected to be visible to Asian/Pacific stations, not all of which had disk-based recording units, either Mk5 or PCEVN types (software correlation of the digital data was key to the project). These problems were addressed in the year leading up to the event.

Seventeen stations participated in the Huygens VLBI experiment; although not all had the capability to detect the signal, the phase referencing corrections do of course benefit by all telescopes.

The probe signal is very weak at the Earth and should be correlated in as narrow a bandwidth as possible. Consider that the VLBA telescopes each received about 20 photons per second from Huygens, and yet a fringe is detected on VLBA baselines with 10 second averaging. It may be the most sensitive VLBI observation ever; it works because the noise power is reduced by filtering while the signal power is undiminished.

The iterative procedure is to track the carrier tone detected by the most sensitive telescope and create a Doppler model which is used to filter with ever smaller bandwidths. At the last 

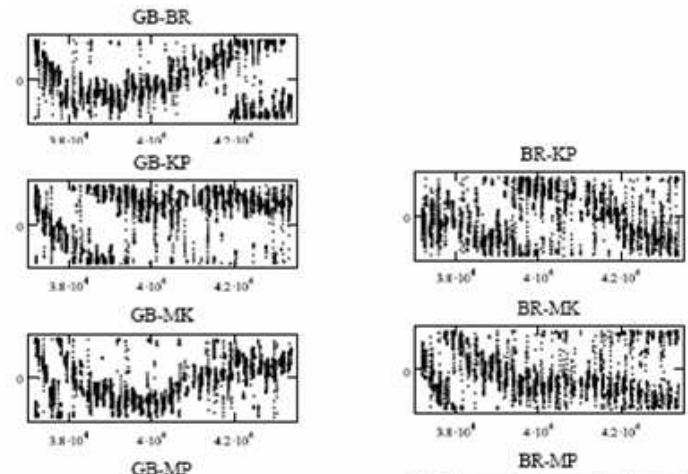

GB.MR
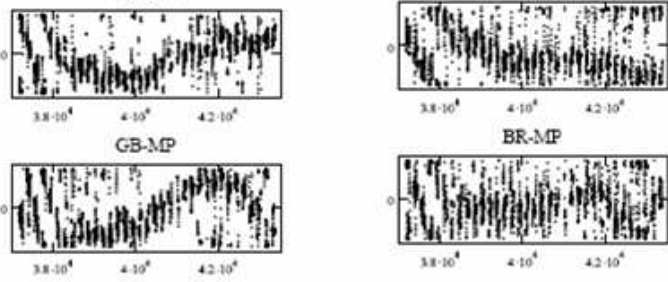

GB-FD

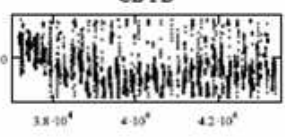

BR.FD

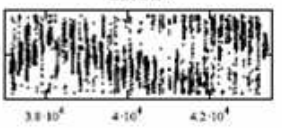

GB-LA

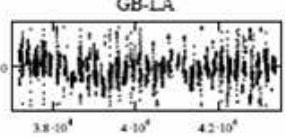

BR-LA

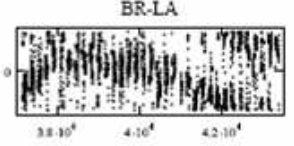

Figure 4: Huygens Probe signal phases on selected baselines during descent.

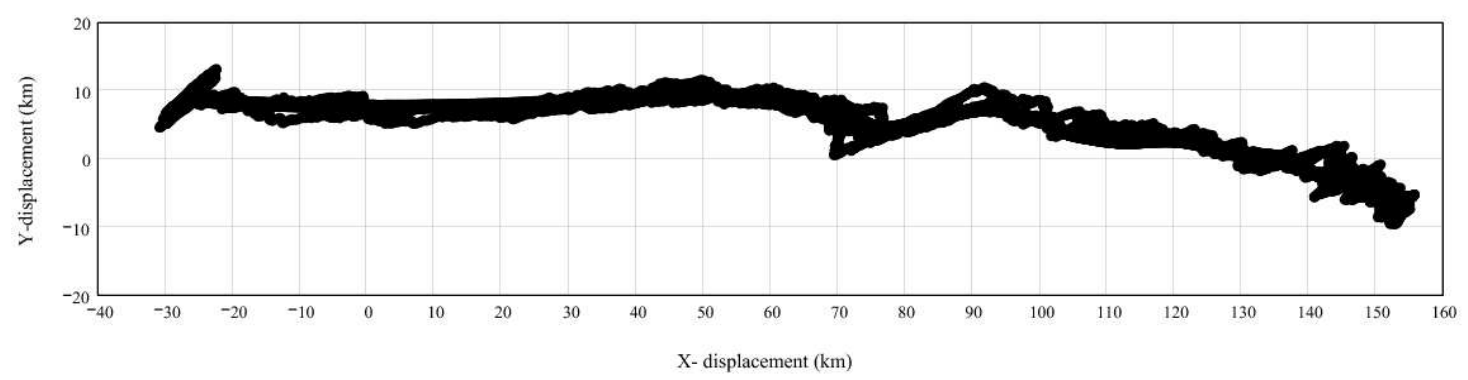

Figure 5: Huygens Probe preliminary trajectory manifold reconstructed from observed phases. The ellipse at start is the $6 \sigma$ error bound. In this trajectory, one point $(0,0)$ is constrained to match the Descent Trajectory Working Group (DTWG) reconstruction.

stage, the globally averaged line is $30 \mathrm{mHz}$ wide. This model is used at all stations, after a frame translation to the geocentre. Phase corrections derived from the reference source with AIPS are applied. Correlation of the filtered Huygens data on a software correlator is then feasible. Figure 4 shows Huygens phases during during descent, and Figure 5 a preliminary trajectory reconstruction.

Standard VLBI delay modeling packages assume infinitely distant sources and Earth-bound observers. Work has been done to include sources in the near-field [6 7]; we are using the VTD package written by Leonid Petrov [8]. Current efforts are in the direction of improving the GPSbased ionospheric gradient corrections, including the observed Doppler profile in self-consistent 
trajectory reconstruction, and deriving error bars on results such as that presented in Figure 5 .

We gratefully acknowledge the assistance of the team of VLBA, ATNF, EVN, IVS and JIVE affiliated staff in the observations and results presented in these proceedings.

\section{References}

[1] B. Bertotti, L. Iess, \& P. Tortora, Nature 425374

[2] D. Doody \& G. Stephan, Basics of Spaceflight Learner's Workbook, JPL D-9774 Rev. A, 1995

[3] P.W. Kinman, DSMS Telecommunications Link Design Handbook, 810-005, Rev. E, 2004

[4] J-P. Lebreton et al, Nature, $\mathbf{4 3 8}, 758$

[5] S.V. Pogrebenko et al, Proc. "Planetary Probe Atmospheric Entry and Descent Trajectory Analysis and Science," ESA SP-544, 2004

[6] S. Kopeikin \& G. Schäfer, Phys. Rev. D, 60, 1999

[7] M. Sekido \& T. Fukushima, IVS NICT-TDC News, No. 24, 11

[8] L. Petrov, private communication 